\title{
A Subset of Patients with Acute Myeloid Leukemia Has Leukemia Cells Characterized by Chemokine Responsiveness and Altered Expression of Transcriptional as well as Angiogenic Regulators
}

\author{
Annette K. Brenner ${ }^{1 *}$, Håkon Reikvam ${ }^{2}$ and Øystein Bruserud ${ }^{1,2}$ \\ ${ }^{1}$ Section for Hematology, Department of Clinical Science, University of Bergen, Bergen, Norway, ${ }^{2}$ Department of Medicine, \\ Haukeland University Hospital, Bergen, Norway
}

OPEN ACCESS

Edited by:

Azzam Maghazachi,

University of Sharjah, United Arab Emirates

Reviewed by:

Daniel Olive,

Inserm UMR 891 Institut Paoli Calmettes, France

Mette D. Hazenberg,

Academic Medical Centre,

Netherlands

*Correspondence:

Annette K. Brenner

annette.brenner@uib.no

Specialty section: This article was submitted to Immunotherapies and Vaccines,

a section of the journal

Frontiers in Immunology

Received: 10 March 2016 Accepted: 11 May 2016

Published: 25 May 2016

Citation:

Brenner AK, Reikvam $\mathrm{H}$ and Bruserud Ø (2016) A Subset of

Patients with Acute Myeloid

Leukemia Has Leukemia Cells

Characterized by Chemokine

Responsiveness and Altered Expression of Transcriptional as well

as Angiogenic Regulators.

Front. Immunol. 7:205.

doi: 10.3389/fimmu.2016.00205
Acute myeloid leukemia (AML) is an aggressive and heterogeneous bone marrow malignancy, the only curative treatment being intensive chemotherapy eventually in combination with allogeneic stem cell transplantation. Both the AML and their neighboring stromal cells show constitutive chemokine release, but chemokines seem to function as regulators of $A M L$ cell proliferation only for a subset of patients. Chemokine targeting is therefore considered not only for immunosuppression in allotransplanted patients but also as a possible antileukemic strategy in combination with intensive chemotherapy or as part of disease-stabilizing treatment at least for the subset of patients with chemokine-responsive AML cells. In this study, we characterized more in detail the leukemia cell phenotype of the chemokine-responsive patients. We investigated primary AML cells derived from 79 unselected patients. Standardized in vitro suspension cultures were used to investigate $\mathrm{AML}$ cell proliferation, and global gene expression profiles were compared for chemokine responders and non-responders identified through the proliferation assays. CCL28-induced growth modulation was used as marker of chemokine responsiveness, and 38 patients were then classified as chemokine-responsive. The effects of exogenous CCL28 (growth inhibition/enhancement/no effect) thus differed among patients and was also dependent on the presence of exogenous hematopoietic growth factors as well as constitutive AML cell cytokine release. The effect of CCR1 inhibition in the presence of chemokine-secreting mesenchymal stem cells also differed among patients. Chemokine-responsive AML cells showed altered expression of genes important for (i) epigenetic transcriptional regulation, particularly lysine acetylation; (ii) helicase activity, especially DExD/H RNA helicases; and (iii) angioregulatory proteins important for integrin binding. Thus, chemokine responsiveness is part of a complex

Abbreviations: AML, acute myeloid leukemia; CLL, chronic lymphocytic leukemia; cpm, counts per minute; ELISA, enzymelinked immunosorbent assay; FAB, French-American-British; Flt3, FMS-like tyrosine kinase 3; Flt3L, Flt3 ligand; GM-CSF, granulocyte macrophage colony-stimulating factor; GO, gene ontology; GPCR, G-protein-coupled receptor; GVHD, graft vs. host disease; NPM1, nucleophosmin; SCF, stem cell factor; SNF/SW1, sucrose non-fermentable/SWItch. 


\begin{abstract}
AML cell phenotype with regard to extracellular communication and transcriptional regulation. Chemokine targeting in chemokine-responsive patients may thereby alter AML cell trafficking and increase their susceptibility toward antileukemic treatment, e.g., conventional chemotherapy or targeting of other phenotypic characteristics of the chemokine-responsive cells.
\end{abstract}

Keywords: acute myeloid leukemia, chemokine, CCL28, integrin, RNA, epigenetic, helicase

\section{INTRODUCTION}

Acute myeloid leukemia (AML) is an aggressive disease characterized by infiltration of malignant myeloblasts in the bone marrow; the only curative treatment is intensive chemotherapy potentially in combination with allogeneic stem cell transplantation (1). However, such intensive treatment is not applicable for elderly or unfit younger patients due to an unacceptable risk of severe toxicity and treatment-related mortality; immunological complications are important causes of the transplant-related mortality that can be due to acute graft vs. host disease (GVHD) as well as severe infections due to the immunocompromised state of patients with chronic GVHD (1).

Several strategies for targeted therapy in AML are now considered $(2,3)$. One possibility is to target the AML-supporting communication between AML cells and their neighboring stromal cells that mediate growth support through their release of soluble mediators, including several chemokines (4-11). Chemokines are involved in this communication; they may function as growth regulators or be important for keeping the leukemic cells in their permissive microenvironment and thereby render them less susceptible to antileukemic therapy. However, in vitro studies suggest that chemokines function as growth regulators in leukemic hematopoiesis only for a subset of AML patients, and a wide range of both CCL and CXCL chemokines can then modulate leukemia cell proliferation (4). One of these chemokines is CCL28 (4) that is released by non-leukemic bone marrow stromal cells, and that preserves the functional integrity of normal hematopoietic progenitor cells (12) through binding to the G-protein-coupled receptors (GPCRs) CCR3 and CCR10 (13-15). CCR3 is a promiscuous receptor, which can bind several ligands in addition to CCL28, whereas CCR10 can only bind CCL27 and CCL28 (16).

Our previous studies have identified a subset of patients whose AML cells show altered proliferation in the presence of exogenous chemokines, and the aim of the present study was to give a broader and more detailed characterization of the AML cell phenotype for these chemokine-responsive patients. First, chemokineresponsive patients show growth modulation in the presence of several chemokines, including CCL28. We therefore used CCL28 responsiveness to identify the chemokine-responsive subset among 79 unselected patients, and because CCL28 is important in normal hematopoiesis, we in addition wanted to characterize both the effects of exogenous CCL28 and chemokine receptor inhibition in leukemic hematopoiesis as parts of our phenotype studies. Second, the phenotype of the chemokine-responsive patient subset was further characterized by comparison of global gene expression profiles for chemokine-responsive and non-responsive patients. A more detailed characterization of this phenotype would be necessary in order to design clinical studies and decide optimal clinical use of targeted therapy in this subset of AML patients.

\section{MATERIALS AND METHODS}

\section{AML Patients and AML Cell Preparation}

The study was conducted in accordance with the Declaration of Helsinki, and the protocol was approved by the local Ethics Committee (Regional Ethics Committee III, University of Bergen). Samples were collected after written informed consent. AML blasts were derived from 79 consecutive patients ( 34 females and 45 males; median age 67 years with range $18-87$ years). Six patients had AML relapse (Table 1) and 11 patients had acquired AML secondary to previous hematological disease (10 patients) or chemotherapy (1 patient). Cytogenetic analyses were available for 71 patients; 9 patients had favorable, 6 patients intermediate, 15 patients adverse, and 41 patients normal cytogenetics, respectively. Our selection of patients and the methods for preparation (gradient separation alone) and characterization of AML cells have been described in detail previously (17).

Prior to the study, we decided to include 80 unselected patients (one patient was left out due to technical reasons); based on a previous study (4), we would then expect to identify at least 25 chemokine responders. This number would be sufficient for statistical comparisons of functional characteristics as well as global gene expression profiles.

The study included only patients with a high percentage $(>80 \%)$ and a high absolute number $\left(>15 \times 10^{9} / \mathrm{L}\right)$ of leukemia cells among the peripheral blood leukocytes (18). A cell population, including $>95 \%$ AML cells, could then be prepared by density gradient separation alone (19). Cells were cryopreserved and stored in liquid nitrogen until used in the experiments (19).

\section{Functional In Vitro Characterization of Primary Human AML Cells}

All cultures of AML cell alone were prepared in serum-free medium (Stem Span, Stem Cell Technologies, Vancouver, BC, Canada), and all recombinant cytokines were supplied by PeproTech (Rocky Hill, NJ, USA). All exogenous cytokines were added at $20 \mathrm{ng} / \mathrm{mL}$, i.e., corresponding to an excess of the added cytokine. Our methods for flow-cytometric characterization of AML cell viability (20), spontaneous and cytokine-dependent proliferation in suspension cultures determined by ${ }^{3} \mathrm{H}$-thymidine incorporation $(4,17)$, constitutive cytokine release (4), and analysis of AML cell viability and proliferation $\left({ }^{3} \mathrm{H}\right.$-thymidine 
TABLE 1 | Clinical and biological characteristics of the 79 unselected patients admitted to our hospital for AML treatment and included in the present study.

\begin{tabular}{|c|c|c|c|c|c|}
\hline \multicolumn{2}{|c|}{ Patient characteristics } & \multicolumn{2}{|l|}{ Cell morphology } & \multicolumn{2}{|l|}{ Cell genetics } \\
\hline Age & & FAB classification & & Cytogenetics ${ }^{a}$ & \\
\hline Median (years) & 67 & $\mathrm{MO}$ & 6 & Favorable & 9 \\
\hline \multirow[t]{2}{*}{ Range (years) } & $18-87$ & M1 & 21 & Intermediate & 6 \\
\hline & & M2 & 11 & Normal & 41 \\
\hline Gender & & M3 & 2 & Adverse & 15 \\
\hline Females & 34 & M4 & 18 & n.d. & 8 \\
\hline \multirow[t]{2}{*}{ Males } & 45 & M5 & 15 & & \\
\hline & & n.d. & 6 & Flt3 mutations & \\
\hline \multicolumn{2}{|l|}{ Secondary AML } & & & ITD & 28 \\
\hline MDS & 7 & CD34 receptor & & Wild-type & 37 \\
\hline Chemotherapy & 1 & Negative $(\leq 20 \%)$ & 21 & n.d. & 14 \\
\hline \multirow[t]{2}{*}{$\mathrm{CM}(\mathrm{M}) \mathrm{L}$} & 3 & Positive (>20\%) & 53 & & \\
\hline & & n.d. & 5 & NPM1 mutations & \\
\hline \multirow[t]{3}{*}{ AML relapse } & 6 & & & Mutated & 26 \\
\hline & & & & Wild-type & 40 \\
\hline & & & & n.d. & 13 \\
\hline
\end{tabular}

ane of the patients had cytogenetic abnormalities with different prognostic impact; inv(16) is associated with a favorable prognosis and +8 with an adverse prognosis.

Inv(16) is regarded to have the strongest impact and therefore to neutralize the negative impact of +8 , the patient was classified as having a favorable karyotype.

${ }^{b}$ One of the patients has an additional point mutation at D835.

n.d., not determined.

incorporation) in transwell cocultures with bone marrow mesenchymal stromal cells [(MSC); MSC24539 purchased from Lonza, Cambrex BioScience, Walkersville, MD, USA] $(8,9)$ have been described in detail previously. CCL28 levels were determined by enzyme-linked immunosorbent assay (ELISA) analysis (R\&D Systems, Abingdon, UK), the minimal detectable level being $45 \mathrm{pg} / \mathrm{mL}$. CXCL2 levels were also determined by ELISA analysis, whereas the levels of the other chemokines were investigated by Luminex analyses (R\&D Systems). The combined CCR1 and CCR3 antagonist J113863 (R\&D Systems) was used at a final concentration of $1.5 \mu \mathrm{M}$ for chemokine receptor inhibition.

\section{RNA Preparation and Analysis of Global Gene Expression}

All microarray data were performed using the Illumina iScan Reader, which is based upon fluorescent detection of biotinlabeled cRNA; $300 \mathrm{ng}$ total RNA from each sample was reversibly transcribed, amplified, and labeled with Biotin-16-UTP using the Illumina TotalPrep RNA Amplification Kit (Applied Biosystems/Ambion, Foster City, CA, USA). Amount and quality of the biotin-labeled cRNA were controlled both by NanoDrop spectrophotometer and Agilent 2100 Bioanalyzer, before $750 \mathrm{ng}$ of biotin-labeled cRNA was hybridized to the HumanHT-12 V4 Expression BeadChip according to the manufacturer's instructions. The chip targets 47,231 probes derived primarily from genes in the NCBI RefSeq database (Release 38).

\section{Bioinformatical and Statistical Analyses}

Bioinformatical analyses were performed using the J-Express 2012 software (MolMine AS, Bergen, Norway). For hierarchical clustering of the cytokine secretion, all values were median-normalized and $\log (10)$-transformed. Complete linkage and Pearson correlation were used as linkage method and distance measurement, respectively. The statistical analyses were performed with the IBM Statistical Package for the Social Sciences (SPSS) version 23 (Chicago, IL, USA). The Mann-Whitney U-test was used to compare value distributions among different patient groups, and $\chi^{2}$ tests (Pearson's $\chi^{2}$ test and the likelihood ratio) were used to determine correlations between different categories. Additionally, Kendall's tau- $b$ correlation tests were performed to determine the agreement between cytokine expression levels in cell supernatants and the mRNA levels of these cytokines. $P$-values $<0.05$ were regarded as statistically significant.

\section{RESULTS}

\section{Identification of a Chemokine-Responsive Patient Subset: Exogenous CCL28 Does Not Alter Spontaneous In Vitro Apoptosis but Modulates AML Cell Proliferation for a Subset of Patients}

There was a wide variation among the 79 AML patients with regard to the viability of the leukemic cells after $40 \mathrm{~h}$ of in vitro culture in medium alone (median viability $36 \%$, range $2-81 \%$ ). The presence of $20 \mathrm{ng} / \mathrm{mL}$ exogenous CCL28 during culture did not significantly alter AML cell viability (median viability 35\%, range 3-78\%) when comparing the overall results (data not shown). Thus, exogenous CCL28 does not have any major effect on the regulation of spontaneous in vitro apoptosis for primary human AML cells.

Proliferation was analyzed using the ${ }^{3} \mathrm{H}$-thymidine incorporation assay, where the nuclide was added after 6 days and nuclear activity assayed $24 \mathrm{~h}$ later. A median value of at least 1,000 counts per minute $(\mathrm{cpm})$ in triplicate cultures was defined as detectable proliferation, whereas a significant alteration of ${ }^{3} \mathrm{H}$-thymidine incorporation was defined as (i) an increase/decrease corresponding to at least $20 \%$ of the control culture, and the absolute value of this change being at least $2,000 \mathrm{cpm}$ for patients with detectable proliferation or alternatively, (ii) a change from detectable to undetectable proliferation or vice versa $(4,17)$. We first compared AML cell proliferation for cultures prepared in medium alone and medium supplemented with $20 \mathrm{ng} / \mathrm{mL}$ of CCL 28 for all 79 patients. The proliferation in medium alone varied among the 79 patients (range $<1,000-9,191 \mathrm{cpm}$ ), and only 17 patients showed detectable autocrine (i.e., spontaneous) proliferation. Exogenous CCL28 increased the proliferation for 14 of the 79 patients compared with the corresponding control cultures prepared in medium alone, whereas decreased proliferation was seen for 3 patients.

\section{The Chemokine-Responsive AML Cell Phenotype: CCL28-Associated Growth Modulation Depends On the Local Cytokine Network}

We investigated the effect of CCL28 on growth factor-dependent proliferation of primary human AML cells for 56 patients that represent a consecutive and thereby unselected subset of the 79 
patients examined in the autocrine proliferation studies described above. The effect of CCL28 was tested in suspension cultures prepared either in medium alone or medium supplemented with exogenous granulocyte macrophage colony-stimulating factor (GM-CSF), stem cell factor (SCF), or FMS-like tyrosine kinase 3 ligand (Flt3L). A detectable proliferative response for at least one of these three growth factors was detected for 42 of the 56 patients, and the results for these patients are summarized in the hierarchical cluster analysis presented in Figure 1 (the analysis also includes the effect of CCL28 for cells cultured in medium alone, see above). We defined a significant alteration as a difference between the CCL28-containing and the corresponding control culture as either (i) a change from/to undetectable levels or (ii) a difference corresponding to an absolute value of at least 2,000 cpm and in addition corresponding to $>20 \%$ of the respective control culture. It can be seen that exogenous CCL28 had divergent effects on AML cell proliferation depending on the local cytokine network. When using these definitions, we observed a significant growth inhibition for 21 and a growth enhancement for 3 out of the 42 patients in the presence of GM-CSF. In contrast, in the presence of Flt3L, significant growth enhancement was seen for 11 patients, and growth inhibition was observed for 3 patients. Finally, CCL28 had generally weaker effects in the presence of SCF; significant inhibition was seen only for six patients and enhancement for two patients. To summarize, CCL28 can alter the proliferation of primary human AML cells for a considerable number of patients, this effect is highly dependent on the local cytokine network and the presence of hematopoietic growth factors, and exogenous CCL28 altered either autocrine or cytokine-dependent (GM-CSF, Flt3L, and SCF) proliferation for 36 of the 56 patients.

CCL28 can bind to the CCR3 and CCR10 receptors (13-15). We used our global gene expression profiles (see below) to compare the expression of CCR3 and CCR10, but we could not detect any significant difference in receptor expression between patients with and without CCL28-associated growth modulation (data not shown). These observations suggest that the differences in chemokine responsiveness between patients is not caused by different regulation of CCR3/CCR10 expression at the mRNA level.

\section{Additional Phenotypic Characteristics of Chemokine-Responsive AML Cells: Constitutive CCL28 Release by AML Cells Is Detected for a Minority of Patients, Showing Weak Association with the Release of Other Chemokines and No Association with Chemokine \\ Responsiveness}

The constitutive release of 11 chemokines was investigated for all 79 patients $\left(2 \times 10^{6}\right.$ cells $/ \mathrm{mL}, 48 \mathrm{~h}$ of in vitro culture). Detectable CCL28 release was observed for only 18 patients (median supernatant level $126 \mathrm{pg} / \mathrm{mL}$, range 49-812 pg/mL), whereas the other chemokines were released for most patients (Table 2). When comparing the overall results, no significant correlation was seen between detectable CCL28 release and cell viability (i.e., spontaneous in vitro apoptosis), differentiation [morphological signs

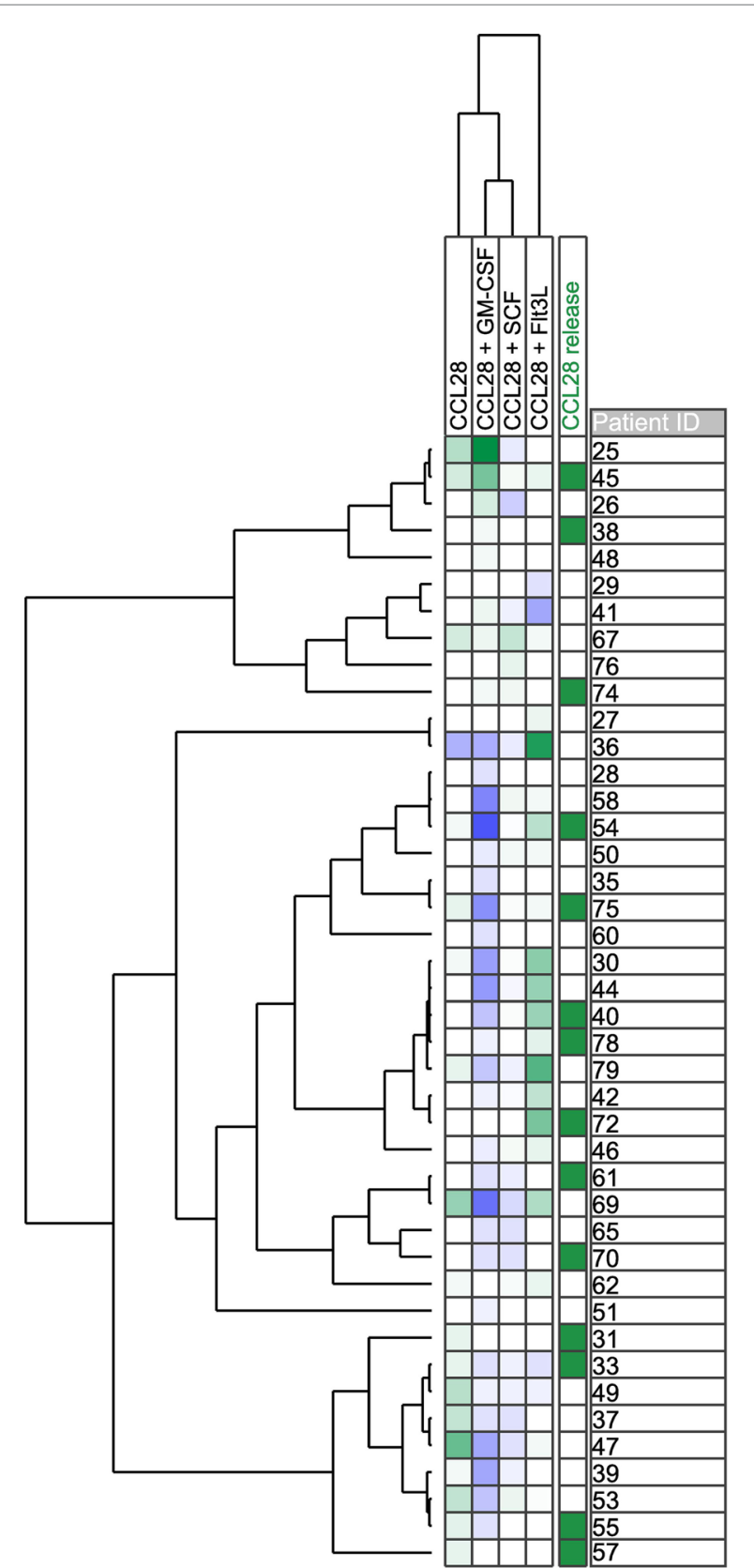

Distance metrics: Pearson Correlation Linkage: COMPLETE

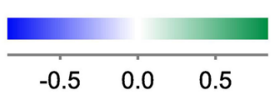

FIGURE 1 | CCL28-associated growth modulation in primary human AML cells; hierarchical clustering analysis of the effects of exogenous CCL28 on the spontaneous and GM-CSF/SCF/FIt3L-dependent proliferation of AML blasts derived from 56 unselected patients. $A M L$ cells were cultured in medium alone or medium supplemented with exogenous GM-CSF, SCF, or Flt3L; for all four types of culture, we compared proliferation for cells cultured with and without exogenous CCL28. All cytokines were tested at a final concentration of $20 \mathrm{ng} / \mathrm{mL}$. For 14 patients, undetectable proliferation (corresponding to a median incorporation of $<1,000 \mathrm{cpm}$ for triplicate determinations) was detected for all cultures; for this reason, we only present the results for the remaining 42 patients. The leukemic cells were cultured in suspension cultures, and the proliferation was measured as ${ }^{3} \mathrm{H}$-thymidine incorporation after 7 days of culture. 
TABLE 2 | Constitutive chemokine release by primary human AML cells; a summary of the results for the 79 unselected patients included in the present study.

\begin{tabular}{lccl} 
Chemokine & $\begin{array}{c}\text { \# patients with detectable } \\
\text { release }\end{array}$ & $\begin{array}{c}\text { Median conc. } \\
\mathbf{( p g / m L})\end{array}$ & $\begin{array}{l}\text { Range } \\
\text { (pg/mL) }\end{array}$ \\
\hline CCL2 & 67 & 110 & n.d.-9,807 \\
CCL3 & 79 & 200 & 119 to $>30,000$ \\
CCL4 & 79 & 92 & $21-11,744$ \\
CCL5 & 79 & 39 & $7.4-2,481$ \\
CCL28 & 18 & $n . d$. & n.d.-812 \\
CXCL1 & 79 & 79 & $41-22,772$ \\
CXCL2 & 69 & 16 & n.d.-11,594 \\
CXCL5 & 78 & 95 & n.d. to $>14,500$ \\
CXCL8 & 79 & 535 & 0.3 to $>18,500$ \\
CXCL10 & 79 & 12 & $1.4-24,642$ \\
CXCL11 & 79 & 83 & $25-246$
\end{tabular}

The levels were determined in the culture supernatants after $48 \mathrm{~h}$ of in vitro culture for the primary AML cells, the supernatant levels of 11 chemokines were determined, and the results are presented as the number of patients with detectable release, the median level for all 79 patients, and the variation range. CCL28 is highlighted.

n.d., not detected, i.e., below detection limit.

according to French-American-British (FAB) classification, expression of the CD34 stem cell marker], karyotype, and Flt3 or NPM1 mutations (data not shown). Finally, there was no significant association between constitutive CCL28 release and spontaneous/autocrine in vitro AML cell proliferation, and constitutive CCL28 release was seen both for patients with and without CCL28-associated growth modulation.

We have previously investigated the constitutive chemokine release for a consecutive patient group, and in the present study, we also determined the supernatant levels for 10 additional chemokines commonly released by primary human AML cells at relatively high levels (79 patients). The chemokine levels for each individual patient was median-normalized and $\log (10)$ transformed prior to Pearson clustering in J-Express. The analysis is presented in Figure 2. Several chemokines were then grouped together in defined subsets. Our previous studies have shown that there are three different chemokine subsets characterized by close clustering and significant correlations between supernatant levels (4); this was also confirmed by our present clustering analysis of constitutive chemokine release: (i) CXCL10/11 clustered close to CCL5; (ii) CCL2/CXCL1/5/8 clustered close to each other; whereas (iii) CXCL2 was the only chemokine tested out of the five members of the third cluster and did not cluster close to CCL28 (4). CCL28 as well as CCL5 clustered as outliers without any strong association with other members of the main chemokine clusters.

It can be seen from Figure 2 that the patients could be divided into four main subsets/clusters; the two upper subsets generally showed high constitutive chemokine release. CCL28-induced growth inhibition for cultures prepared with GM-CSF and SCF was significantly more frequent in this high chemokine release group (15 and 6 out of the upper 42 patients, respectively) compared with the low-release group (6/37 and 0/37; likelihood ratios $=0.023$ and 0.003 , respectively). This observation further supports our hypothesis from the studies of CCL28 effects in the presence of exogenous cytokines (GM-CSF, Flt3L, and SCF, see above); the final effects of CCL28 on AML cell proliferation depends on the local cytokine network determined by the constitutive cytokine release by the leukemic cells (Figure 2) and exogenous cytokines (i.e., cytokines not released by AML cells).

\section{Additional Phenotypic Characterization of AML Cells: Normal Blood Cell Counts at Diagnosis Do Not Differ among Patients with and without Constitutive CCL28 Release}

Bone marrow failure with pancytopenia in peripheral blood is common at the time of diagnosis for AML patients (1). Furthermore, CCL28 is a growth and survival factor for normal hematopoietic cells (12), and for this reason we compared peripheral blood hemoglobin levels and platelet counts at the time of diagnosis for patients with and without constitutive CCL28 release by their AML cells. We could not detect any significant difference between the two groups with regard to $\mathrm{Hb}$ levels or platelet counts at the time of diagnosis (data not shown).

\section{Additional Phenotypic Characterization of Chemokine-Responsive Patients: Chemokine Receptor Blocking Has Antileukemic Effects Only for Certain Patients}

We investigated the effects of endogenous chemokines using an in vitro model, where primary human AML cells and normal bone marrow MSCs derived from a healthy donor were separated by a semipermeable membrane during coculture (9); the MSCs can then influence AML cell proliferation only through their release of soluble mediators. We tested the effects of the combined CCR 1 (ligands being CCL2/3/5/7/14/15/16/23/28) and CCR3 (ligands being CCL5/7/8/11/13/15/24/28) antagonist J113863 on AML cell viability and proliferation; viability was investigated by a flowcytometric assay and proliferation as ${ }^{3} \mathrm{H}$-thymidine incorporation $(8,9)$. We investigated this pharmacological effect for primary AML cells derived from five CCL28/chemokine responders. The inhibitor had divergent effects on AML cell proliferation during coculture with chemokine-releasing MSCs; for one patient, growth inhibition was seen (median proliferation corresponding to 9,457 vs. 3,502 cpm), for three other patients, increased proliferation was observed ( 21,323 vs. $26,253 \mathrm{cpm}, 10,934$ vs. $19,713 \mathrm{cpm}$, and 55,257 vs. $74,956 \mathrm{cpm}$, respectively), and for the last patient, only a minor effect of CCL28 was seen $(4,800$ vs. $4,392 \mathrm{cpm})$ in the cocultures. These experiments show that chemokines mediate divergent effects on AML cell proliferation that differs among the chemokine responders also, when tested in the presence of cytokine/chemokine-releasing bone marrow MSCs.

\section{Additional Phenotypic Characterization of Chemokine-Responsive Patients: Chemokine Responsiveness Is Associated with Altered Expression of Genes Important for Protein Acetylation, Helicase Activity, and Integrin Binding}

In a previous study, we observed that CCL28 could modulate AML cell proliferation only for a subset of patients (4); this was 


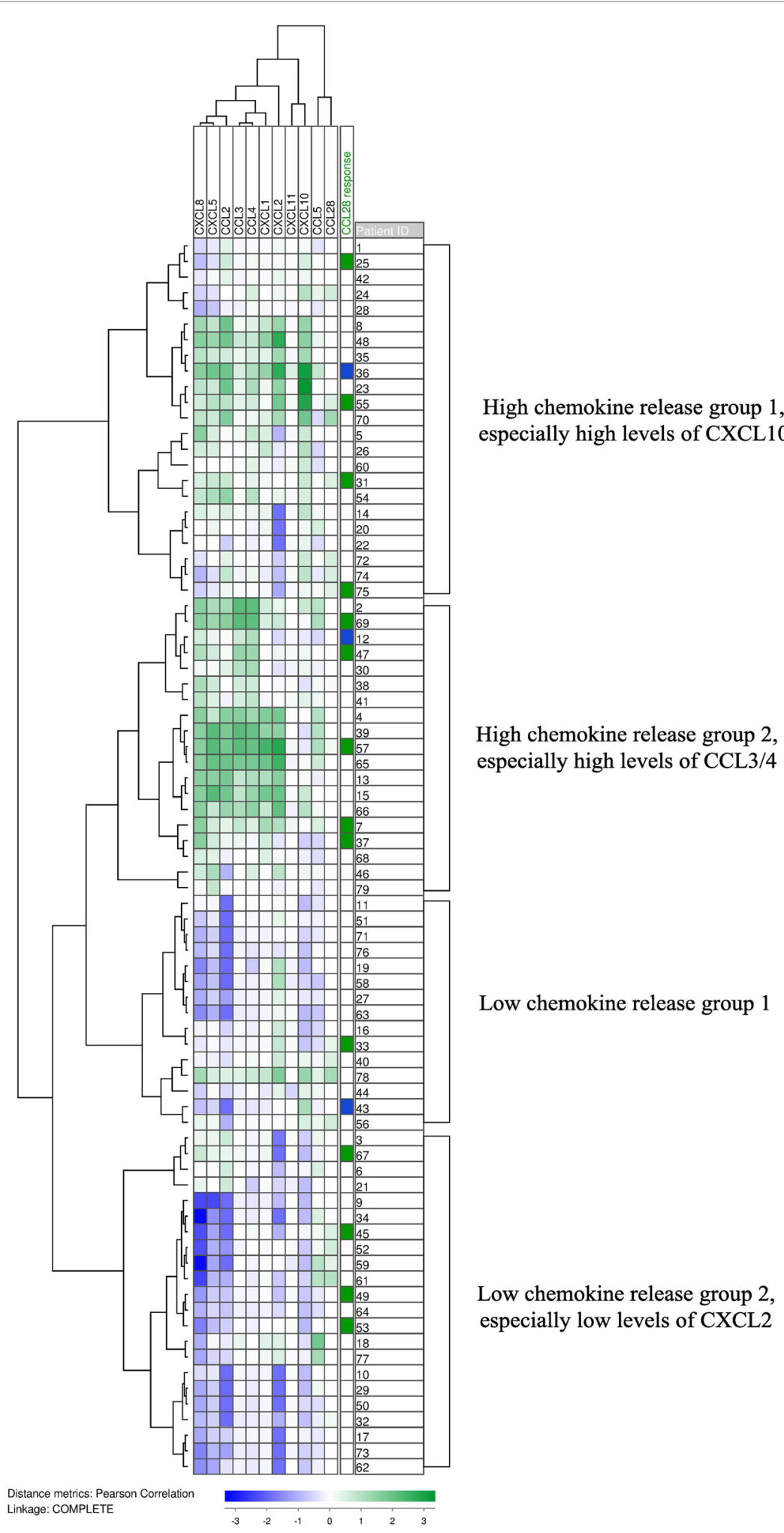

FIGURE 2 | The constitutive chemokine release profile by primary human AML cells. The constitutive release of 11 chemokines, including CCL28, during in vitro culture was examined for primary human AML cells derived from 79 consecutive patients. Chemokine release was determined as the protein level in culture supernatants. Based on the hierarchical clustering analysis, the patients could be divided into four subclusters. CCL28, together with CCL5, formed an out-group and did not correlate with the expression of the other nine chemokines. The column on the right shows the 14 patients whose cells responded with increased proliferation to CCL28 (green) in medium alone without supplementation of exogenous growth factors, and the 3 patients whose blasts showed reduced proliferation in the presence of CCL28 compared to cultures containing only medium (blue). 
also confirmed in our present study (Figure 1). The previous study showed that for this particular patient subset, AML cell proliferation could be modulated not only by CCL2 8 but also by several other chemokines binding to different chemokine receptors (4). Thus, CCL28 growth modulation should be regarded as a marker of a patient subset that, in contrast to other patients, are characterized by chemokine-induced (GPCR-mediated) growth modulation. We then compared the global gene expression profiles for 37 unselected patients; 15 of these patients being classified as responders, according to the definitions used above for the effects of exogenous CCL28 on spontaneous and/or growth factor-dependent (GM-CSF, Flt3L, and SCF) proliferation. However, with regard to the classification of patients as responders/non-responders and the change to/from undetectable from/ to detectable proliferation (i.e., proliferation corresponding to $>1,000 \mathrm{cpm}$ defined as detectable, see above), this definition is arbitrary and four of the seven patients with such a change showed a relatively small absolute difference between cultures with and without exogenous CCL28. Even though there was a change from undetectable to detectable with CCL28, two of these four patients therefore were by chance classified as responders and the two others as non-responders.

We first identified those genes showing a difference between the two patient subsets corresponding to $p<0.001$ and a false discovery rate (FDR) of $<0.01$. The identified genes (21) were included in 20 gene ontology (GO)-terms; a major part of these terms were associated with integrin binding (Table 3, Table S1 in Supplementary Material), protein/lysine/histone acetylation (Table 4, Table S2 in Supplementary Material), or helicase activity (Table 5, Table S3 in Supplementary Material). The term integrin binding included 27 differentially expressed genes; among them were several proteases and angioregulatory mediators, seven genes encoding extracellular matrix molecules, and several adhesion molecules. The large part of the proteins encoded by these genes seems to play a role in the regulation of local angiogenesis, a process important both for the development and chemosensitivity of human AML (8). Integrins may also be important for modulation of intracellular signaling initiated by ligation of the CCR3 chemokine receptor (22). Second, the terms histone acetylation, lysine-protein acetylation, and internal peptidyl lysine acetylation were overlapping and included 48 genes; a large number of them are involved in histone modulation/acetylation and/or in transcriptional regulation (Table 4, Table S2 in Supplementary Material). Finally, the largest group of differentially regulated genes were included in the GO term Helicase Activity (Table 5, Table S3 in Supplementary Material). This group included mainly RNA helicases (e.g., 23 members of the DEAD box family RNA helicases together with several DEAH box family RNA helicases), mini-chromosome maintenance proteins, and members of the sucrose non-fermentable/ SWItch (SNF/SW1) family.

\section{DISCUSSION}

Several recent studies have emphasized the importance of investigating the cancer cell phenotype for understanding carcinogenesis, defining patient subsets in heterogeneous malignancies,
TABLE 3 | Comparison of global gene expression profiles for primary human AML cells with and without chemokine-induced growth modulation - a summary of the differentially expressed genes encoding proteins important for integrin binding (for additional details, see Table $\mathbf{S} 1$ in Supplementary Material).

Classification of genes based on the protein function Angioregulation vascular biology

Proteases

ADAM2, a disintegrin and metallopeptidase (ADAM)

domain 2

ADAM23, ADAM metallopeptidase domain 23

ADAM22, ADAM metallopeptidae domain 22

ADAMTS8, ADAM metallopeptidase with thrombospondin type 1 motif 8

Extracellular matrix molecules

COL3A1, collagen type III alpha 1

COL5A1, collagen type $V$ alpha 1 (vascular stability)

COL16A1, collagen type XVI alpha 1

FBLN5, fibulin 5

LAMA5, laminin alpha 5

LAMB2, laminin beta 2 (angioregulator)

TNN, tenascin N

Soluble mediators involved in angiogenesis

ANGPTL1, angiopoietin-like 1

CYR61, cysteine-rich angiogenic inducer 61

JAM3, junctional adhesion molecule 3 (the soluble form)

WWF, von Willebrand factor

Cell-to-cell and cell-to-matrix adhesion

ICAM3, intercellular adhesion molecule 3 (LFA-1 ligand)

ITGB1BP1, integrin beta 1 binding protein 1

ITGB6, integrin beta 6

JAM3, junctional adhesion molecule 3

THBS4, thrombospondin 4

THY1, Thy-1 cell surface antigen

Other genes encoding proteins involved in angiogenes

EMP2, epithelial membrane protein 2 (integrin modulator)

$\mathrm{KDR}$, kinase insert domain receptor (VEGF receptor)

NMB (neuromedin B)

SOD1, superoxide dismutase 1 , soluble

Other genes

IMPAD1, inositole monophosphatase domain containing 1

OXCT1, 3-oxoacid transferase 1 (mitchondrial metabolism)

and identifying possible therapeutic targets (23-25). The aim of the present study was therefore to characterize the AML cell phenotype more in detail for the subset of patients having chemokine-responsive leukemic cells. Our present observations suggest that these patients respond to exogenous chemokines and combination of chemokine/chemokine receptor targeting either with other targeted therapies (e.g., epigenetic or integrin-targeting) or conventional chemotherapy may therefore be considered especially for the identified patient subset.

Chemokine receptor blockers can be used as an immunosuppressive strategy, e.g., as GVHD therapy in AML patients receiving allogeneic stem cell transplantation (21). Chemokine targeting may thus be used in these patients (i) as a pre-/posttransplant antileukemic strategy with direct effects on the leukemic cells at least for patients with chemokine-responsive cells or (ii) as an immunosuppressive treatment. GVHD has antileukemic effects, and immunosuppression due to severe GVHD will reduce this effect. However, at least for patients with chemokine-responsive 
TABLE 4 | A comparison of the global gene expression profiling of primary human AML cells with and without chemokine-induced growth modulation - a summary of the differentially expressed genes that encode proteins important for histone acetylation (for additional details, see Table S2 in Supplementary Material).

Main classification based on the protein function

Gene identity

Chromatin modulation/histone acetylation and transcriptional

regulation

ACTL6A, CHD9, DMAP1, EPC1, ING3, KAT2A, KAT2B, KIAA1267, MEAF6,

MLL, MYST1, MYST2, MYST4, SRCAP, SMARCA4, TRRAP, YEATS2

Transcriptional regulation only

BRD8, BRPF1, CHD9, GTF3C4, EP400, HCFC1, LDB1, MECP2, MYOD1,

OGT, PHD15, PHD16, PHF17, PHF20, TAF1, TAF1L, TAF6L, TAF15, TCF3

DNA repair

BRCA2, HCFC1, PHF20, TRRAP

Cell cycle regulation

HCFC20

Genes with other or unknown functions

BAT3 (apoptosis), CCDC101, CPA3 (protease), KIAA1310, MBIP, MSL2 USP22

AML cells, the use of chemokine targeting may represent a therapeutic alternative that reduces the GVHD-associated antileukemic reactivity but at the same time has an additional antileukemic effect by itself against residual AML.

Acute myeloid leukemia is a heterogeneous and aggressive disease, and in a previous study of another smaller patient population, we observed that exogenous CCL28 could modulate cytokine-dependent AML cell proliferation for a minority of patients. For this patient subset, CCL28 was one out of several chemokines with growth-modulating effect (4). Several chemokine receptor inhibitors are now available, and this strategy is considered in the treatment of AML both as an AML-directed antileukemic treatment or as an immunosuppressive strategy for patients with immune-mediated complications following allogeneic stem cell transplantation (21). In the present article, we have studied a large group of patients more in detail, and for these patients, the growth modulation seems to be a part of a wider phenotype that also includes altered epigenetic/ transcriptional regulation, RNA metabolism/splicing, and local regulation of angiogenesis. Many chemokines and chemokine receptors show promiscuous receptor/ligand binding, and targeting of single chemokines may therefore have limited effect because a wide range of chemokines are present in the bone marrow microenvironment (9). Combined targeting with different GPCR antagonists or combination with agents that target other aspects of this complex phenotype may then be considered for this particular subset of patients.

CCL28 is a chemokine involved in normal hematopoiesis (12). We now describe that it can also be involved in leukemic hematopoiesis and modulate the growth of primary human AML cells for a subset of patients. However, its final effect depends on the local cytokine network. Growth inhibition was observed in the presence of the exogenous cytokines, SCF and especially GM-CSF, whereas growth enhancement was seen in the presence
TABLE 5 | Comparison of global gene expression profiles of primary human AML cells with and without chemokine-induced growth modulation - a summary of the differentially expressed genes encoding proteins important for helicase activity.

$\begin{aligned} & \text { Classification of genes based } \\ & \text { on the protein structure and/or }\end{aligned}$
function

DEAD box family of proteins (RNA helicases)

DDX4, DDX10 (involved in

leukemogenesis?), DDX17, DDX18

(activated by Myc)

DDX27/50/51/52/54/58/60/60L

DOX19A

$\mathrm{IFIH} 1$

DDX3X/6/28

DEAH box family of proteins

$\mathrm{DH} \times 8 / 9 / 40$

$\mathrm{DH} 29$

WRN (DNA repair?) + +

Mini-chromosome maintenance proteins (genome replication)

MCMDC1 (chromatin modulation)

$\mathrm{MCM} 7 / 9$

MCM8 +

SNF/SW1 family of protein (chromatin remodeling)

ATRX

HLTF

SMARCA2, SMARCA4, SMARCAL1

Other helicases

CHD3 (histone deacetylation)

$\mathrm{CHD} 4$ (histone deacetylation)

CHD6 (chromatin remodeling)

$\mathrm{CHD} 8$ (chromatin remodeling)

CHD9

MOV10

HELZ

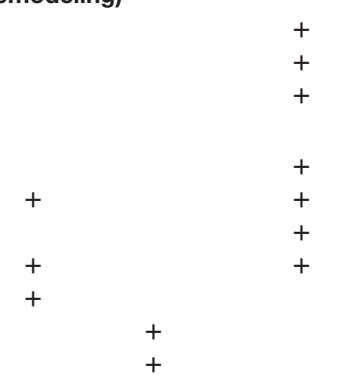

Proteins involved in DNA methylation, chromatin remodeling, and DNA

repair

ASCC3 (DNA repair)

ERCC8 (DNA repair), SHPRH (DNA

repair?), SRCAP (histone remodeling)

Other genes/proteins

EIF4A1

EP400 (protein synthesis?)

GTF2F2

JARID2 (mutations associated with

myeloid malignancies)

PRIC285

RAD54L2

TDRD12

YTHDC2

ZRANB3 (DNA repair?)

Additional characteristics that possibly are important for leukemogenesis are given in parenthesis at the end of the functional description (for additional details, see Table S3 in Supplementary Material). To the right in the figure is indicated whether the proteins are important for the function of DNA, RNA function/metabolism, or transcriptional regulation.

of exogenous Flt3L. Finally, CCL28 can be constitutively released by primary human AML cells and thereby be a part of the cross talk between leukemic cells and their neighboring bone marrow cells, but the ability of constitutive CCL28 release showed no association with autocrine proliferation or the effects of exogenous CCL28. 
We included 79 patients in the present study, and they represent a consecutive and thereby unselected group, except that all of them showed relatively high levels of circulating AML cells. This strategy was used to allow a standardized preparation of highly enriched AML cells by gradient separation alone, thereby avoiding the risk of inducing functional alterations in the cells by more extensive separation procedures (17). The use of this strategy has been discussed previously (17), and our study population does not differ from the overall patient population regarding important biological, clinical, and prognostic parameters (4) (see below).

In our study, we investigated primary AML cells from consecutive/unselected patients. These cell populations have a hierarchical organization with (i) a majority of more mature cells undergoing stress-induced apoptosis during in vitro culture; (ii) an intermediate subset of clonogenic progenitors (often $<1 \%$ ); and (iii) a small minority of AML stem cells $(18,26)$. Studies of the total cell population should still be regarded as relevant despite this organization, especially with regard to antileukemic therapy, because: (i) early morphological disease control with remission induction (i.e., reduction of the total AML cell population) is an important prognostic parameter (1); (ii) clinical studies have shown that biological characteristics of the total AML cell population reflect general leukemic cell characteristics that have prognostic impact and are essential for leukemogenesis and chemosensitivity (26); and (iii) previous studies have also shown that the AML stem cells can be detected in various subsets, usually in the $\mathrm{CD} 34^{+} \mathrm{CD} 38^{-}$subset and also in the $\mathrm{CD} 34^{-}$and $\mathrm{CD} 34^{+} \mathrm{CD} 38^{+}$ subsets, and stem cell characteristics are also reflected in the overall cell population $(18,26)$.

All our methods for cell preparation, in vitro culture, and AML cell characterization have been described in previous methodological investigations. First, our approach with inclusion of only patients with relatively high peripheral blood AML cell counts have been characterized previously when consecutive patients with high blood AML cell counts were compared with the overall consecutive AML patient population (17). The two groups did not differ with regard to other prognostic (i.e., chemosensitivity) parameters, but despite this, one should be careful when generalizing from our results (17). Second, the use of cryopreservation has also been discussed previously, and its effect on AML cell viability has been described in detail $(19,20)$. Third, we used serum-free medium for culture of AML cells (27), the only exception being the cocultures with MSCs, because these mesenchymal cells require an enriched medium (9). Finally, as described above, the AML cell population has a hierarchical organization (26), and our proliferation assay based on ${ }^{3} \mathrm{H}$-thymidine incorporation allows us to analyze only the proliferation of long-term surviving cells able to proliferate after 7 days of culture; these cells represents an enrichment of clonogenic cells (18). Thus, our study is based on well-characterized and standardized methodological strategies.

Chemokine receptor inhibitors are now being developed $(28,29)$. However, patients showing chemokine-dependent growth modulation usually respond to a wide range of chemokines that bind different receptors (4), and both the constitutive chemokine/cytokine release by AML cells and the release by neighboring bone marrow stromal cells will determine the final effect of chemokine receptor binding on AML cell proliferation.
These observations suggest that combined targeting of promiscuous chemokine receptors would be most effective in AML. However, the intracellular signaling downstream to the various chemokine receptors is difficult to predict $(16,22)$, and our present results show that even such broad targeting of a promiscuous receptor has divergent effects for chemokine-responsive patients. It is not known whether combined targeting of the other phenotypic characteristics of these patients will be more effective.

Constitutive CCL28 release was observed for a minority of patients and showed no correlation with autocrine proliferation, chemokine responsiveness, or gene expression profiles. Despite these observations, CCL28 may contribute to leukemogenesis or chemosensitivity through its effect on neighboring stromal cells. Recent studies have demonstrated that CCR3 and CCR10 receptors are expressed by bone marrow stromal cells (30). The bone marrow microenvironment is hypoxic, and studies in experimental models strongly suggest that CCL28 is important for cell trafficking and angiogenesis in hypoxic microenvironments associated with carcinogenesis (31). CCL28 is also released by endothelial cells and may thereby be a part of the cross talk between AML and neighboring stromal cells. Through its release by non-leukemic stromal cells in the bone marrow microenvironment $(7,32)$, CCL28 may still function as a growth factor for remaining normal hematopoietic cells in AML (12). Finally, as discussed below, there may also be an interaction between integrins and CCL28, as the latter promotes integrin-dependent cell adhesion (33).

We investigated the effect of CCR1/CCR3 inhibition (both being promiscuous receptors) in a more physiological model of AML/MSC cocultures; this model was used to study chemokine effects in a local cytokine network with a major influence of bone marrow stromal elements. Our results show that even in a cytokine environment formed by the constitutive cytokine release and the cytokine-mediated cross talk between primary AML and bone marrow MSCs, the CCR1/CCR3-mediated effects on AML cell proliferation are divergent, and the different effects of CCR1/ CCR3 inhibition on AML cell viability and proliferation can be explained by the hierarchical organization of the AML cell population (17). The viability studies then reflect the characteristics of the majority of more mature cells that undergo spontaneous apoptosis during the first days of culture, whereas the proliferation is assayed after several days of culture and reflects the ability of a more immature cell minority capable of maintaining proliferation after several days.

Even though we could not detect effects of chemokine inhibition on AML cell proliferation in vitro, studies in chronic lymphocytic leukemia (CLL) have shown that altered regulation of in vivo cell trafficking can contribute significantly to the antileukemic effects of certain drugs (34-39). The microenvironment is important both for survival and proliferation of CLL cells, and local chemokine release is a part of this support. Furthermore, ibrutinib mediates its antileukemic effect in CLL through direct effects on the CLL cells and through inhibition of chemokine-dependent leukemia cell trafficking, thereby keeping the cells out of their growthenhancing/antiapoptotic microenvironment and making them susceptible to the direct antileukemic effect. Our present study shows that a subset of patients has chemokine-responsive AML 
cells, and similar dual-targeting may be possible in AML. This hypothesis is supported by recent studies; ibrutinib has direct antileukemic effects in human AML (40) and is in addition able to alter chemokine-dependent AML cell migration (41). Our present studies therefore suggest combined targeting of AML cell survival, and migration (i.e., chemokine targeting) should be considered for patients with chemokine-responsive AML cells.

In our studies of global gene expression profiles, we observed that AML cells with CCL28-induced growth modulation (i.e., chemokine-responsive) showed an altered expression of genes included in the GO-terms lysine acetylation/integrins/helicases. As discussed above, there is a functional interaction between CCL28 and integrins, but there is also a biological interaction between the integrin system and lysine acetylation. First, histone acetylation is important for regulation of gene expression $(2,42)$, including the expression of integrins in human AML cells (43). Second, lysine acetylation can also alter integrin expression indirectly; HOX transcription factors are important for regulation of integrin gene expression, and acetylation is then important in the regulation of HOX activity both through effects on the mRNA expression of these transcription factors and through modulation of their transcription factor activity by posttranscriptional modulation of HOX proteins $(44,45)$. Third, experimental studies suggest that integrins can mediate intracellular signaling that alters histone acetylation and thereby alter the proliferation of malignant cells $(46,47)$. Through this signaling, integrins can also regulate gene expression in human myeloid cells through the activation of several transcription factors (48). Finally, acetylation of microtubules is important for regulation of the cell surface density of integrins (49). Thus, through their interactions with extracellular matrix and neighboring cells, integrins mediate bidirectional signaling, i.e., inside-out and outside-in signaling $(50,51)$, and lysine acetylation is important at several steps of this process.

Helicases are also important for transcriptional and translational regulation and thereby seem to represent a functional overlap with lysine acetylation. First, a major part of the helicases with altered expression are members of the DEAD box family of RNA helicases, they belong to the helicase superfamily 2 together with the DEAH helicases and are often referred to as $\mathrm{DExD} / \mathrm{H}$ box RNA helicases $(52,53)$. The helicases should be regarded as multifunctional proteins important in regulation of transcription, ribosome biogenesis, translation, and RNA decay/ storage/metabolism; they also have ATPase activity and can function as metabolic sensors (53-55). The helicase DDX10 can be involved in leukemogenesis (56). Second, the mini-chromosome maintenance complex forms an important part of the replication machinery, but its loading to chromatin at sites distant from replicating DNA strongly suggests that it has additional functions, possibly as transcriptional regulators (57). Third, SWI/SNF is a multisubunit chromatin-remodeling complex important for regulation of cell cycle progression, apoptosis, differentiation, genomic instability, and DNA repair (58). The function of this molecular complex in AML seems to differ from other malignancies, and it is considered as a possible therapeutic target in human AML $(59,60)$.

\section{CONCLUSION}

Chemokines have growth-modulating effects on the AML cells only for a minority of patients; and these patients are then responsive to several chemokines. However, this chemokine responsiveness is only a part of a more extensive leukemia cell phenotype that includes several partly interacting cellular functions, i.e., altered extracellular communication (chemokines/ integrins/acetylation) and transcriptional regulation (histone acetylation, helicases) compared with the other non-responsive AML cells. Furthermore, the direct effects of chemokine targeting on primary human AML cells are difficult to predict because they depend both on differences among patients and on the overall local cytokine network; this is probably true both if chemokine targeting is tried as an antileukemic strategy or as an approach to treat immune-mediated complications in allotransplanted AML. A careful evaluation of the AML cells will possibly be important to avoid the risk of leukemia enhancement and thereby increased relapse risk; this would probably be important if the treatment is tried both as an antileukemic and an immunosuppressive strategy. A possible approach to avoid this risk may be combined targeting of other aspects of this complex phenotype. However, based on the lessons from recent studies in CLL where chemokines are important for the supportive function of neighboring stromal cells by keeping the CLL cells within their permissive microenvironment, one would emphasize that AML cells from chemokine-responsive patients should be further tested in relevant in vivo models (e.g., patient-derived xenografts) to see whether chemokine targeting alters AML cell trafficking/homing and thereby increases their chemosensitivity.

\section{AUTHOR CONTRIBUTIONS}

$\varnothing \mathrm{B}$ conceived and designed the experiments; $\mathrm{AB}$ performed the experiments; $\mathrm{AB}$ and $\mathrm{HR}$ analyzed the data; and $\varnothing \mathrm{B}$ and $\mathrm{AB}$ wrote the paper.

\section{ACKNOWLEDGMENTS}

The study received financial support from the Norwegian Cancer Society, Helse-Vest, and the University of Bergen. The technical assistance of Kristin P. Rye and Karen Marie Hagen is highly appreciated.

\section{FUNDING}

Helse-Vest funding: 911788. The Norwegian Cancer Society funding: 62370, 62371, and 100933.

\section{SUPPLEMENTARY MATERIAL}

The Supplementary Material for this article can be found online at http://journal.frontiersin.org/article/10.3389/fimmu. 2016.00205 


\section{REFERENCES}

1. Döhner H, Estey EH, Amadori S, Appelbaum FR, Buchner T, Burnett AK, et al. Diagnosis and management of acute myeloid leukemia in adults: recommendations from an international expert panel, on behalf of the European LeukemiaNet. Blood (2010) 115:453-74. doi:10.1182/blood-2009-07-235358

2. Shafer D, Grant S. Update on rational targeted therapy in AML. Blood Rev (2016). doi:10.1016/j.blre.2016.02.001

3. Stein EM, Tallman MS. Emerging therapeutic drugs for AML. Blood (2016) 127:71-8. doi:10.1182/blood-2015-07-604538

4. Bruserud Ø, Ryningen A, Olsnes AM, Stordrange L, Øyan AM, Kalland KH, et al. Subclassification of patients with acute myelogenous leukemia based on chemokine responsiveness and constitutive chemokine release by their leukemic cells. Haematologica (2007) 92:332-41. doi:10.3324/haematol.10148

5. Bruserud Ø, Ryningen A, Wergeland L, Glenjen NI, Gjertsen BT. Osteoblasts increase proliferation and release of pro-angiogenic interleukin 8 by native human acute myelogenous leukemia blasts. Haematologica (2004) 89:391-402.

6. Dahlberg A, Delaney C, Bernstein ID. Ex vivo expansion of human hematopoietic stem and progenitor cells. Blood (2011) 117:6083-90. doi:10.1182/ blood-2011-01-283606

7. Gao B, Sun W, Wang X, Jia X, Ma B, Chang Y, et al. Whole genome expression profiling and screening for differentially expressed cytokine genes in human bone marrow endothelial cells treated with humoral inhibitors in liver cirrhosis. Int J Mol Med (2013) 32:1204-14. doi:10.3892/ijmm.2013.1495

8. Hatfield K, Ryningen A, Corbascio M, Bruserud Ø. Microvascular endothelial cells increase proliferation and inhibit apoptosis of native human acute myelogenous leukemia blasts. Int J Cancer (2006) 119:2313-21. doi:10.1002/ ijc. 22180

9. Reikvam H, Brenner AK, Hagen KM, Liseth K, Skrede S, Hatfield KJ, et al. The cytokine-mediated crosstalk between primary human acute myeloid cells and mesenchymal stem cells alters the local cytokine network and the global gene expression profile of the mesenchymal cells. Stem Cell Res (2015) 15:530-41. doi:10.1016/j.scr.2015.09.008

10. Walasek MA, van Os R, de Haan G. Hematopoietic stem cell expansion: challenges and opportunities. Ann N Y Acad Sci (2012) 1266:138-50. doi:10.1111/j.1749-6632.2012.06549.x

11. Sison EA, Brown P. The bone marrow microenvironment and leukemia: biology and therapeutic targeting. Expert Rev Hematol (2011) 4:271-83. doi:10.1586/ehm.11.30

12. Karlsson C, Baudet A, Miharada N, Soneji S, Gupta R, Magnusson M, et al. Identification of the chemokine CCL28 as a growth and survival factor for human hematopoietic stem and progenitor cells. Blood (2013) 121(383842):S1-15. doi:10.1182/blood-2013-02-481192

13. Gong L, Wilhelm RS. CCR3 antagonists: a survey of the patent literature. Expert Opin Ther Pat (2009) 19:1109-32. doi:10.1517/13543770903008544

14. Willems LI, Ijzerman AP. Small molecule antagonists for chemokine CCR3 receptors. Med Res Rev (2010) 30:778-817. doi:10.1002/med.20181

15. Xiong N, Fu Y, Hu S, Xia M, Yang J. CCR10 and its ligands in regulation of epithelial immunity and diseases. Protein Cell (2012) 3:571-80. doi:10.1007/ s13238-012-2927-3

16. Steen A, Larsen Ø, Thiele S, Rosenkilde MM. Biased and g protein-independent signaling of chemokine receptors. Front Immunol (2014) 5:277. doi:10.3389/ fimmu.2014.00277

17. Bruserud $\varnothing$, Hovland R, Wergeland L, Huang TS, Gjertsen BT. Flt3-mediated signaling in human acute myelogenous leukemia (AML) blasts: a functional characterization of Flt3-ligand effects in AML cell populations with and without genetic Flt3 abnormalities. Haematologica (2003) 88:416-28.

18. Bruserud Ø, Gjertsen BT, Foss B, Huang TS. New strategies in the treatment of acute myelogenous leukemia (AML): in vitro culture of aml cells - the present use in experimental studies and the possible importance for future therapeutic approaches. Stem Cells (2001) 19:1-11. doi:10.1634/stemcells.19-1-1

19. Gjertsen BT, Øyan AM, Marzolf B, Hovland R, Gausdal G, Døskeland SO, et al. Analysis of acute myelogenous leukemia: preparation of samples for genomic and proteomic analyses. J Hematother Stem Cell Res (2002) 11:469-81. doi:10.1089/15258160260090933

20. Ryningen A, Ersvær E, Øyan AM, Kalland KH, Vintermyr OK, Gjertsen BT, et al. Stress-induced in vitro apoptosis of native human acute myelogenous leukemia (AML) cells shows a wide variation between patients and is associated with low BCL-2:Bax ratio and low levels of heat shock protein 70 and 90. Leuk Res (2006) 30:1531-40. doi:10.1016/j.leukres.2006.02.014

21. Kittan NA, Hildebrandt GC. The chemokine system: a possible therapeutic target in acute graft versus host disease. Curr Top Microbiol Immunol (2010) 341:97-120. doi:10.1007/82_2010_23

22. Shamri R, Young KM, Weller PF. PI3K, ERK, p38 MAPK and integrins regulate CCR3-mediated secretion of mouse and human eosinophil-associated RNases. Allergy (2013) 68:880-9. doi:10.1111/all.12163

23. Gad H, Koolmeister T, Jemth AS, Eshtad S, Jacques SA, Strom CE, et al. MTH1 inhibition eradicates cancer by preventing sanitation of the dNTP pool. Nature (2014) 508:215-21. doi:10.1038/nature13181

24. Helleday T. Cancer phenotypic lethality, exemplified by the non-essential MTH1 enzyme being required for cancer survival. Ann Oncol (2014) 25:1253-5. doi:10.1093/annonc/mdu158

25. Helleday T, Eshtad S, Nik-Zainal S. Mechanisms underlying mutational signatures in human cancers. Nat Rev Genet (2014) 15:585-98. doi:10.1038/ nrg3729

26. Eppert K, Takenaka K, Lechman ER, Waldron L, Nilsson B, van Galen P, et al. Stem cell gene expression programs influence clinical outcome in human leukemia. Nat Med (2011) 17:1086-93. doi:10.1038/nm.2415

27. Bruserud Ø, Gjertsen BT, von Volkman HL. In vitro culture of human acute myelogenous leukemia (AML) cells in serum-free media: studies of native AML blasts and AML cell lines. J Hematother Stem Cell Res (2000) 9:923-32. doi:10.1089/152581600750062372

28. Allegretti M, Cesta MC, Garin A, Proudfoot AE. Current status of chemokine receptor inhibitors in development. Immunol Lett (2012) 145:68-78. doi:10.1016/j.imlet.2012.04.003

29. Proudfoot AE, Power CA, Schwarz MK. Anti-chemokine small molecule drugs: a promising future? Expert Opin Investig Drugs (2010) 19:345-55. doi:10.1517/13543780903535867

30. Rankin SM. Chemokines and adult bone marrow stem cells. Immunol Lett (2012) 145:47-54. doi:10.1016/j.imlet.2012.04.009

31. Facciabene A, Peng X, Hagemann IS, Balint K, Barchetti A, Wang LP, et al. Tumour hypoxia promotes tolerance and angiogenesis via CCL28 and T(reg) cells. Nature (2011) 475:226-30. doi:10.1038/nature10169

32. Chen Z, Kim SJ, Essani AB, Volin MV, Vila OM, Swedler W, et al. Characterising the expression and function of CCL28 and its corresponding receptor, CCR10, in RA pathogenesis. Ann Rheum Dis (2015) 74:1898-906. doi:10.1136/ annrheumdis-2013-204530

33. Miles A, Liaskou E, Eksteen B, Lalor PF, Adams DH. CCL25 and CCL28 promote alpha4 beta7-integrin-dependent adhesion of lymphocytes to MAdCAM-1 under shear flow. Am J Physiol Gastrointest Liver Physiol (2008) 294:G1257-67. doi:10.1152/aipgi.00266.2007

34. Burger JA. Targeting the microenvironment in chronic lymphocytic leukemia is changing the therapeutic landscape. Curr Opin Oncol (2012) 24:643-9. doi:10.1097/CCO.0b013e3283589950

35. Burger JA, Montserrat E. Coming full circle: 70 years of chronic lymphocytic leukemia cell redistribution, from glucocorticoids to inhibitors of B-cell receptor signaling. Blood (2013) 121:1501-9. doi:10.1182/blood-2012-08-452607

36. Chen SS, Chang BY, Chang S, Tong T, Ham S, Sherry B, et al. BTK inhibition results in impaired CXCR4 chemokine receptor surface expression, signaling and function in chronic lymphocytic leukemia. Leukemia (2016) 30:833-43. doi:10.1038/leu.2015.316

37. Hartmann EM, Rudelius M, Burger JA, Rosenwald A. CCL3 chemokine expression by chronic lymphocytic leukemia cells orchestrates the composition of the microenvironment in lymph node infiltrates. Leuk Lymphoma (2016) 57:563-71. doi:10.3109/10428194.2015.1068308

38. Herishanu Y, Katz BZ, Lipsky A, Wiestner A. Biology of chronic lymphocytic leukemia in different microenvironments: clinical and therapeutic implications. Hematol Oncol Clin North Am (2013) 27:173-206. doi:10.1016/j. hoc.2013.01.002

39. Ponader S, Chen SS, Buggy JJ, Balakrishnan K, Gandhi V, Wierda WG, et al. The Bruton tyrosine kinase inhibitor PCI-32765 thwarts chronic lymphocytic leukemia cell survival and tissue homing in vitro and in vivo. Blood (2012) 119:1182-9. doi:10.1182/blood-2011-10-386417

40. Zaitseva L, Murray MY, Shafat MS, Lawes MJ, MacEwan DJ, Bowles KM, et al. Ibrutinib inhibits SDF1/CXCR4 mediated migration in AML. Oncotarget (2014) 5:9930-8. doi:10.18632/oncotarget.2479 
41. Rotin LE, Gronda M, MacLean N, Hurren R, Wang X, Lin FH, et al. Ibrutinib synergizes with poly(ADP-ribose) glycohydrolase inhibitors to induce cell death in AML cells via a BTK-independent mechanism. Oncotarget (2016) 7:2765-79. doi:10.18632/oncotarget.6409

42. Pastore F, Levine RL. Epigenetic regulators and their impact on therapy in acute myeloid leukemia. Haematologica (2016) 101:269-78. doi:10.3324/ haematol.2015.140822

43. Mahlknecht U, Schonbein C. Histone deacetylase inhibitor treatment downregulates VLA-4 adhesion in hematopoietic stem cells and acute myeloid leukemia blast cells. Haematologica (2008) 93:443-6. doi:10.3324/ haematol.11796

44. Taniguchi Y. Hox transcription factors: modulators of cell-cell and cell-extracellular matrix adhesion. Biomed Res Int (2014) 2014:591374. doi:10.1155/2014/591374

45. Zhu LH, Sun LH, Hu YL, Jiang Y, Liu HY, Shen XY, et al. PCAF impairs endometrial receptivity and embryo implantation by down-regulating beta3-integrin expression via HOXA10 acetylation. J Clin Endocrinol Metab (2013) 98:4417-28. doi:10.1210/jc.2013-1429

46. Inaba J, McConnell EJ, Davis KR. Lunasin sensitivity in non-small cell lung cancer cells is linked to suppression of integrin signaling and changes in histone acetylation. Int J Mol Sci (2014) 15:23705-24. doi:10.3390/ijms151223705

47. Rose JL, Huang H, Wray SF, Hoyt DG. Integrin engagement increases histone $\mathrm{H} 3$ acetylation and reduces histone $\mathrm{H} 1$ association with DNA in murine lung endothelial cells. Mol Pharmacol (2005) 68:439-46. doi:10.1124/ mol.104.010876

48. Shi C, Simon DI. Integrin signals, transcription factors, and monocyte differentiation. Trends Cardiovasc Med (2006) 16:146-52. doi:10.1016/j. tcm.2006.03.002

49. Joo EE, Yamada KM. MYPT1 regulates contractility and microtubule acetylation to modulate integrin adhesions and matrix assembly. Nat Commun (2014) 5:3510. doi:10.1038/ncomms4510

50. Futosi K, Fodor S, Mocsai A. Reprint of Neutrophil cell surface receptors and their intracellular signal transduction pathways. Int Immunopharmacol (2013) 17:1185-97. doi:10.1016/j.intimp.2013.11.010

51. Gehler S, Ponik SM, Riching KM, Keely PJ. Bi-directional signaling: extracellular matrix and integrin regulation of breast tumor progression. Crit Rev Eukaryot Gene Expr (2013) 23:139-57. doi:10.1615/CritRevEukarGeneExpr .2013006647
52. Cencic R, Pelletier J. Throwing a monkey wrench in the motor: targeting DExH/D box proteins with small molecule inhibitors. Biochim Biophys Acta (2013) 1829:894-903. doi:10.1016/j.bbagrm.2013.01.008

53. Fuller-Pace FV. DExD/H box RNA helicases: multifunctional proteins with important roles in transcriptional regulation. Nucleic Acids Res (2006) 34:4206-15. doi:10.1093/nar/gkl460

54. Hooper C, Hilliker A. Packing them up and dusting them off: RNA helicases and mRNA storage. Biochim Biophys Acta (2013) 1829:824-34. doi:10.1016/j. bbagrm.2013.03.008

55. Linder P, Fuller-Pace FV. Looking back on the birth of DEAD-box RNA helicases. Biochim Biophys Acta (2013) 1829:750-5. doi:10.1016/j. bbagrm.2013.03.007

56. Yassin ER, Abdul-Nabi AM, Takeda A, Yaseen NR. Effects of the NUP98DDX10 oncogene on primary human CD34+ cells: role of a conserved helicase motif. Leukemia (2010) 24:1001-11. doi:10.1038/leu.2010.42

57. Das M, Singh S, Pradhan S, Narayan G. MCM Paradox: abundance of eukaryotic replicative helicases and genomic integrity. Mol Biol Int (2014) 2014:574850. doi:10.1155/2014/574850

58. Biegel JA, Busse TM, Weissman BE. SWI/SNF chromatin remodeling complexes and cancer. Am J Med Genet C Semin Med Genet (2014) 166C:350-66. doi:10.1002/ajmg.c.31410

59. Buscarlet M, Krasteva V, Ho L, Simon C, Hebert J, Wilhelm B, et al. Essential role of BRG, the ATPase subunit of BAF chromatin remodeling complexes, in leukemia maintenance. Blood (2014) 123:1720-8. doi:10.1182/ blood-2013-02-483495

60. Hohmann AF, Vakoc CR. A rationale to target the SWI/SNF complex for cancer therapy. Trends Genet (2014) 30:356-63. doi:10.1016/j.tig.2014.05.001

Conflict of Interest Statement: The authors declare that the research was conducted in the absence of any commercial or financial relationships that could be construed as a potential conflict of interest.

Copyright (c) 2016 Brenner, Reikvam and Bruserud. This is an open-access article distributed under the terms of the Creative Commons Attribution License (CC BY). The use, distribution or reproduction in other forums is permitted, provided the original author(s) or licensor are credited and that the original publication in this journal is cited, in accordance with accepted academic practice. No use, distribution or reproduction is permitted which does not comply with these terms. 